\title{
The problem of anomalous dilepton yield in relativistic heavy-ion collisions
}

\author{
Alexander Andrianov* \\ St.Petersburg State University \\ E-mail: Sashaandrianov@gmail.com
}

Vladimir A. Andrianov

St.Petersburg State University

E-mail: W.andrianodrambler.ru

\section{Domenec Espriu and Xumeu Plannels}

Departament d'ECM and ICCUB-Institut de Ciencies del Cosmos, University of Barcelona,

Spain

E-mail: espriudecm.ub.edu, vumeudicc.ub.es

The recent data on abnormal excess of dileptons in central heavy ion collisions are surveyed. We propose a novel explanation for this dilepton excess observed in dense/hot nuclear matter at invariant masses below $1 \mathrm{GeV}$. We argue that the presence of local parity breaking (LPB) due to a slowly varying time-dependent isosinglet and/or isotriplet pseudoscalar condensate may substantially modify the dispersion relation of photons and vector mesons propagating in such a medium, resulting in a large excess of $l^{+} l^{-}$with respect to the common theoretical predictions. Various experimental options to prove or falsify this effect are discussed.

The XIXth International Workshop on High Energy Physics and Quantum Field Theory, QFTHEP2010 September 08-15, 2010

Golitsyno, Moscow, Russia

\footnotetext{
${ }^{*}$ Speaker.
} 


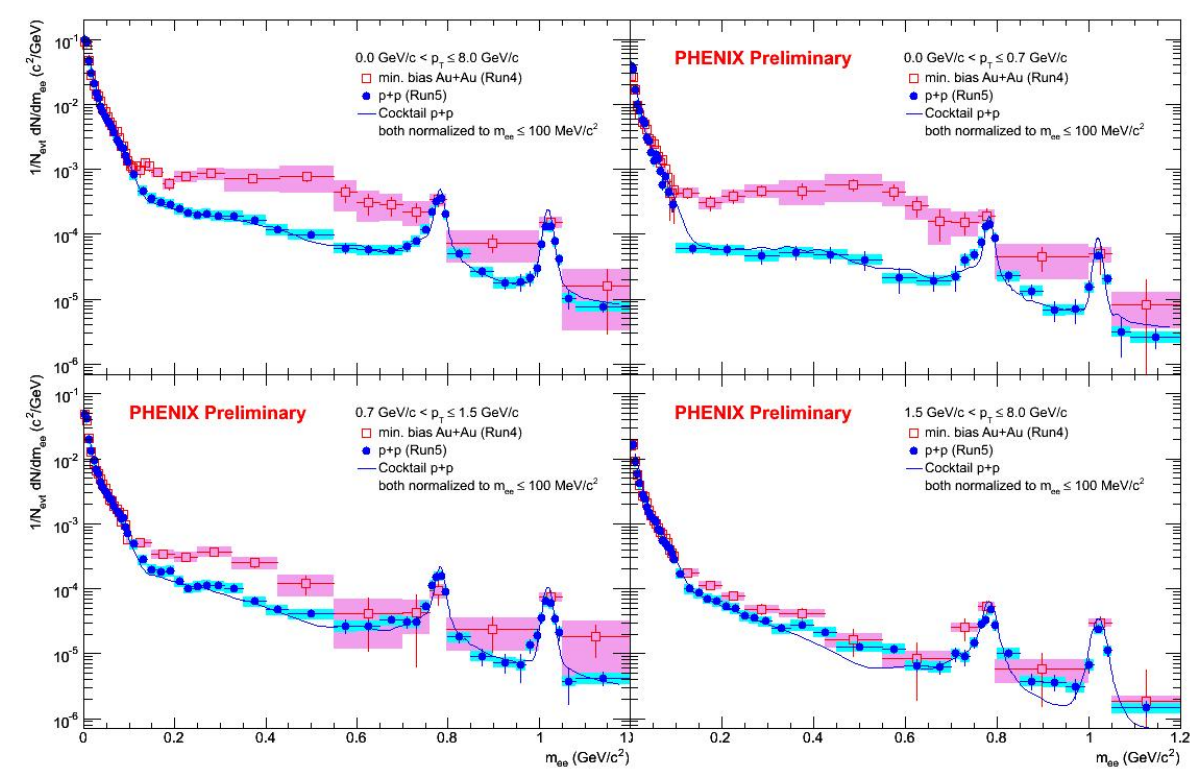

Figure 1: Abnormal dilepton excess in $A u+A u$ ion collisions measured by the PHENIX collaboration [प]: dependence on different ranges of transverse momenta

\section{Introductory review}

The possibility of parity $(P)$ breaking via pseudoscalar condensation into a large-scale classical field for sufficiently high temperatures and/or chemical potentials has been within the scope of several studies during last decades. The expected area of its manifestation is, first of all, the hot and dense nuclear matter produced in heavy ion collisions at intermediate and high energies ( and also in neutron/quark stars). At finite baryon density its appearance was conjectured long ago in [W] as a result of pion-nucleon interaction. However this scenario presupposes a space dependent pion condensate which has not been justified well in low energy nuclear physics [[]]. A more sophisticated model for pion condensation based on interference of the pion and excited pion configurations has been elaborated in [3] and it predicts neutral pion condensation in infinite nuclear matter (or creation of large-scale pion classical field in finite nuclear fireballs) at intermediate baryon densities several times higher than the normal ones. However these opportunities may not be realizable for high temperatures and low baryon chemical potentials. On the contrary, the possibility of $(C) P$ parity breaking arises in meta-stable nuclear bubbles created in hot nuclear matter [䧃] as a result of large fluctuations of isosinglet topological charge which is dual to a nontrivial chiral chemical potential. Thus in either dense or hot baryon matter one anticipates (local) parity breaking. The actual question is on how to detect this phenomenon in heavy ion collisions at intermediate and high energies and disentangle it from other nuclear reactions. Certainly, the particle probes weakly depending on strong interactions are most favorable to do this job.

Recently several experiments in heavy ion collisions have indicated an abnormal yield of lepton pairs of invariant mass $<1 \mathrm{GeV}$ in the region of small rapidities and moderate transversal

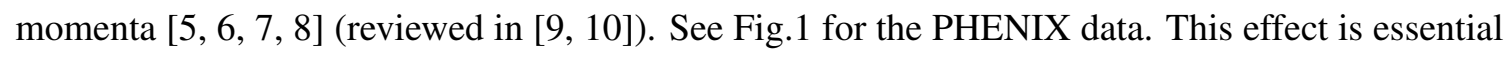
only for collisions that are central or nearly central (see Fig.D). Most studies observe $e^{+} e^{-}$pairs 


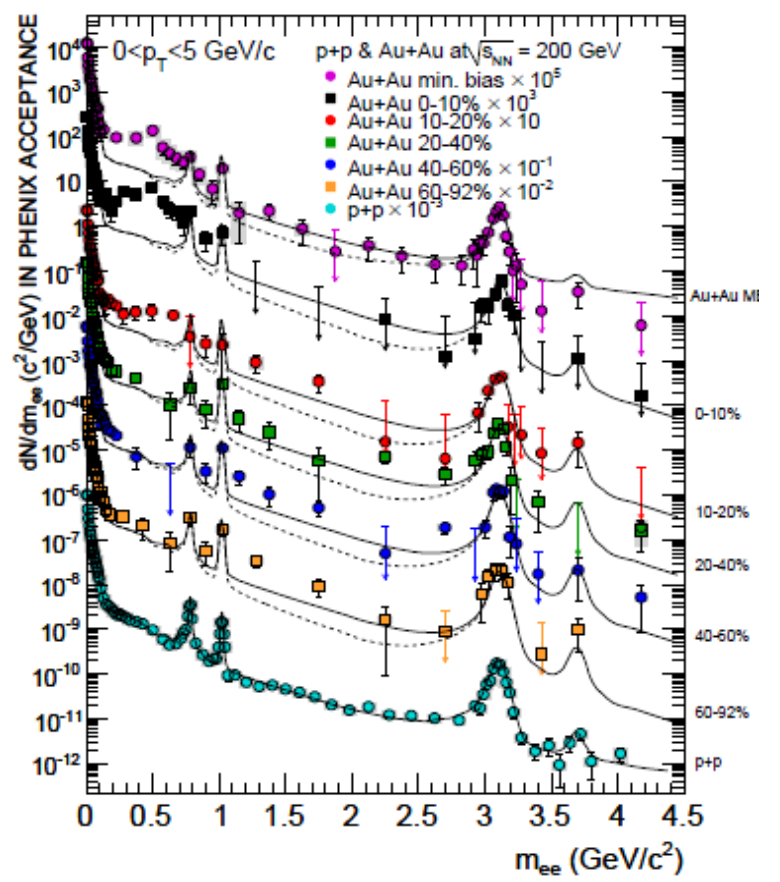

TABLE I The enhancement factor, defined as the ratio between the measured yield and the expected yield for $0.15<m_{c e}<0.75 \mathrm{GeV} / c^{2}$, for different centrality bins.

\begin{tabular}{cc}
\hline \hline Centrality & Enhancement $( \pm$ stat \pm syst \pm model $)$ \\
\hline $00-10 \%$ & $7.6 \pm 0.5 \pm 1.5 \pm 1.5$ \\
$10-20 \%$ & $3.2 \pm 0.4 \pm 0.1 \pm 0.6$ \\
$20-40 \%$ & $1.4 \pm 1.3 \pm 0.02 \pm 0.3$ \\
$40-60 \%$ & $0.8 \pm 0.3 \pm 0.03 \pm 0.2$ \\
$60-92 \%$ & $1.5 \pm 0.3 \pm 0.001 \pm 0.3$ \\
Min. Bias & $4.7 \pm 0.4 \pm 1.5 \pm 0.9$ \\
\hline \hline
\end{tabular}

Figure 2: Abnormal dilepton excess in $A u+A u$ ion collisions measured by the PHENIX collaboration [ $[$ ] . The centrality dependence is clearly demonstrated and the very effect is visible mostly for $M_{e e}<1 \mathrm{GeV}$.

but dimuon pairs have also been found to be produced in excess above the dimuon threshold [1], 团].

From comparison to proton-proton and proton-nucleus collisions it has been well established that such an enhancement is undoubtedly a nuclear medium effect [Q]. For the energies accessible at GSI (of few GeV per nucleon in HADES experiments [ [ $[$ ]) with light nuclei the above effect was partially interpreted as being due to enhanced $\eta$ meson production in proton-neutron scattering [Ш]]. For the higher energies accessible at CERN SPS and BNL RHIC of order of $100 \mathrm{GeV}$ per nucleon, in CERES, HELIOS/3, NA60 [[]] and, especially, in PHENIX [U] (see, Fig. (B]) experiments, the abnormal dilepton yield has not been yet explained at full extent by any mechanisms known in hadron phenomenology [Q, 四].

Following $[\square]$ we shall divide the range of invariant masses into high (beyond $3.2 \mathrm{GeV}$ ), low (LMR, below $1.2 \mathrm{GeV}$ ) and intermediate (between $1.2 \mathrm{GeV}$ and $3.2 \mathrm{GeV}$ ). In the LMR mostly the different Dalitz processes and the $\rho$ meson decay dominate in the dilepton production and the enhancement could be, in principle, explained by possible modification of meson properties in nu-

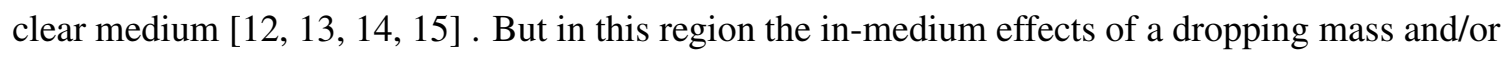
broadening resonance happen to be insufficient to explain the spectacular dilepton enhancement by a 4 to 7 factor, depending on $p_{T}$ and centrality, observed in PHENIX [U] experiments. A similar abnormal enhancement has been seen also in NA60 [可] experiments with a better (but not complete) saturation by thermal in-medium effects [एँ3]].

In our talk we give a radically different explanation of this enhancement[ए]]. We suggest that the effect may be a manifestation of local parity breaking (LPB) in colliding nuclei due to 


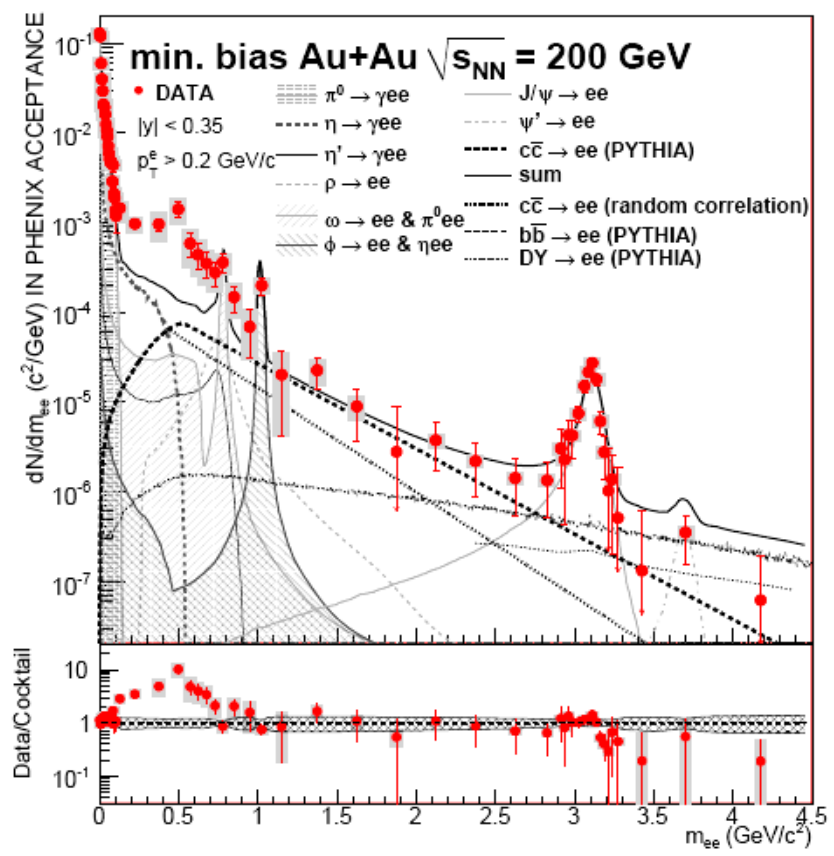

Figure 3: Abnormal dilepton excess in $A u+A u$ ion collisions measured by the PHENIX collaboration [D]: comparison of the hadronic (normalized to $p p$ scattering) and (computed) charm cocktail.

generation of pseudoscalar, isosinglet or neutral isotriplet, classical background whose magnitude depends on the dynamics of the collision. It has been suggested that such a background could appear as the result of large-scale fluctuation of topological charge leading for non-central collisions to the so-called Chiral Magnetic Effect (CME)[四] which has been studied by lattice QCD simulations [ए]] and seemingly detected in the STAR experiments on RHIC [ए8], although the issue is not yet fully settled. It may be also related to pseudoscalar domain walls [एव]. However the fact that the observed dilepton excess is absent for peripheral collisions (where the CME should be more visible) and maximized in cental collisions makes us believe that it may rather correspond to the ephemeral formation of a locally bona-fide thermodynamic phase where parity is broken, a possibility that has been argued for in [B]].

\section{Time dependent pseudoscalar field triggers abnormal production of dileptons}

It has been shown in [Z] that a pseudoscalar field slowly evolving in time changes drastically the electromagnetic properties of the vacuum. In particular an energetic in-medium photon propagating in this background may decay on-shell into dileptons. This same mechanism extended to vector mesons is proposed here as the source for abnormal yield of $l^{+} l^{-}$pairs in the LMR, i.e. in the range $0.15 \mathrm{GeV}<M_{e e}<1 \mathrm{GeV}$ for $e^{+} e^{-}$production or $0.2 \mathrm{GeV}<M_{\mu \mu}<1 \mathrm{GeV}$ for $\mu^{+} \mu^{-}$ production, for centrality $0 \div 20 \%$ and for moderate $p_{T}<1 \mathrm{GeV}$ [Q, 目]. Herein we will concentrate in the region around the $\rho$ and $\omega$ resonant contribution.

We shall assume that a time dependent but approximately spatially homogeneous background of a pseudoscalar field $a(t)$ is induced at the densities reached in heavy ion collisions and we will 
define a 4-vector related, $\hat{\zeta}_{\mu} \simeq \partial_{\mu} a \simeq \delta_{0 \mu} \hat{\zeta}$. $a(t)$ could be either isosinglet or isotriplet or even a mixture of the two, $\hat{\zeta}=\zeta \mathbf{I}+\zeta_{3} \tau_{3}$, but detailed calculations will be presented for the case of constant isosinglet background $\zeta$ only. A more detailed discussion of this ansatz is postponed to the end of this section.

The appropriate framework to describe electromagnetic interactions of hadrons at low energies is the Vector Dominance Model (VDM)[ㄱ], 2] ] containing the lightest vector mesons $\rho_{0}$ and $\omega$ in the $S U$ (2) flavor sector. We do not include $\phi$ meson, as its typical mean free path $\sim 40 \mathrm{fm}$ makes it insensitive to medium effects. Quark-meson interactions are described by

$$
\begin{aligned}
& \mathscr{L}_{\text {int }}=\bar{q} \gamma_{\mu} V^{\mu} q ; \quad V_{\mu} \equiv-e A_{\mu} Q+\frac{1}{2} g_{\omega} \omega_{\mu} \mathbf{I}+g_{\rho} \rho_{\mu} \frac{\tau_{3}}{2}, \\
& \left(V_{\mu, a}\right) \equiv\left(A_{\mu}, \omega_{\mu}, \rho_{\mu} \equiv\left(\rho_{0}\right)_{\mu}\right),
\end{aligned}
$$

where $Q=\frac{\tau_{3}}{2}+\frac{1}{6} \mathbf{I}, g_{\omega} \simeq g_{\rho} \equiv g \simeq 6$. These values are extracted from vector meson decays. The Maxwell and mass terms are

$$
\begin{aligned}
& \mathscr{L}_{\text {kin }}=-\frac{1}{4}\left(F_{\mu v} F^{\mu v}+\omega_{\mu v} \omega^{\mu v}+\rho_{\mu v} \rho^{\mu v}\right) \\
& \mathscr{L}_{\text {mass }}=m_{V}^{2} \operatorname{tr}\left(V_{\mu} V^{\mu}\right)=\frac{1}{2} V_{\mu, a} m_{a b}^{2} V_{b}^{\mu}, \\
& m_{a b}^{2}=m_{V}^{2}\left(\begin{array}{ccc}
\frac{10 e^{2}}{9 g^{2}} & -\frac{e}{3 g} & -\frac{e}{g} \\
-\frac{e}{3 g} & 1 & 0 \\
-\frac{e}{g} & 0 & 1
\end{array}\right), \operatorname{det}\left(m^{2}\right)=0,
\end{aligned}
$$

where $m_{V}^{2}=m_{\rho}^{2}=2 g_{\rho}^{2} f_{\pi}^{2} \simeq m_{\omega}^{2}$. This matrix reflects the VMD relations at the quark level [[1], [1]]. Finally, in a pseudoscalar time-dependent background the Lagrangian contains a parity-odd ChernSimons (CS) term

$$
\begin{aligned}
& \mathscr{L}_{C S}(k)=-\frac{1}{4} \varepsilon^{\mu v \rho \sigma} \operatorname{tr}\left\{\hat{\zeta}_{\mu} V_{v}(x) V_{\rho \sigma}(x)\right\} \\
& =\frac{1}{2} \operatorname{tr}\left\{\hat{\zeta} \varepsilon_{j k l} V_{j} \partial_{k} V_{l}\right\}=\frac{1}{2} \zeta \varepsilon_{j k l} V_{j, a} N_{a b} \partial_{k} V_{l, b},
\end{aligned}
$$

which additionally mixes photons and vector mesons due to LPB. For isosinglet pseudoscalar background $e^{2} \hat{\zeta}=\frac{9}{5} \zeta \mathbf{I}$, and the mixing matrix reads

$$
N_{a b} \simeq\left(\begin{array}{ccc}
1 & -\frac{3 g}{10 e} & -\frac{9 g}{10 e} \\
-\frac{3 g}{10 e} & \frac{9 g^{2}}{10 e^{2}} & 0 \\
-\frac{9 g}{10 e} & 0 & \frac{9 g^{2}}{10 e^{2}}
\end{array}\right), \operatorname{det}(N)=0 .
$$

Remarkably, $N \sim m^{2}$. Simple order-of-magnitude considerations indicate that $\zeta \sim \alpha \tau^{-1} \sim 1 \mathrm{MeV}$, taking the time of formation of pseudoscalar condensate $\tau=1 \mathrm{fm}$ and the value of condensate of order of $f_{\pi}$.

For isotriplet pseudoscalar background $e^{2} \hat{\zeta}=3 \zeta \tau_{3}$, and the corresponding CS matrix takes the form

$$
N_{a b}^{\pi} \simeq\left(\begin{array}{ccc}
1 & -\frac{3 g}{2 e} & -\frac{g}{2 e} \\
-\frac{3 g}{2 e} & 0 & \frac{3 g^{2}}{2 e^{2}} \\
-\frac{g}{2 e} & \frac{3 g}{2 e^{2}} & 0
\end{array}\right), \quad \operatorname{det}\left(N^{\pi}\right)=0
$$


The VMD coefficients in (2.4), (2.5) are obtained from the anomalous Wess-Zumino action [22] and related to the phenomenology of radiative decays of vector mesons [23]. The ratios of matrix elements for isotriplet condensate in ([2.5) are in agreement with the experimental decay constants for the processes $\pi_{0} \rightarrow \gamma \gamma, \quad \omega \rightarrow \pi_{0} \gamma, \quad \rho_{0} \rightarrow \pi_{0} \gamma$ [22] and for the decay $\omega \rightarrow \pi \pi \pi$ [24] taken from [DS]. Likewise the elements in (뎌) can be estimated from the decays $\eta \rightarrow \gamma \gamma, \quad \eta^{\prime} \rightarrow \gamma \gamma, \quad \omega \rightarrow$ $\eta \gamma, \quad \rho_{0} \rightarrow \eta \gamma$ after taking into account the $\eta_{8}-\eta_{0}$ mixing [26]. However, phenomenologically there exists a strong $\eta_{8}-\eta_{0}$ mixing effect finally resolved in the SU(3) flavor scheme [26]. Only the ratio of the decay widths $\omega \rightarrow \eta \gamma, \quad \rho_{0} \rightarrow \eta \gamma$ is little sensitive to the mixing and confirms the off-diagonal elements of (‥4). Here we ignore the above mixing and restrict ourselves to $\mathrm{SU}(2)$.

The mass-shell equations for vector mesons read

$$
\begin{aligned}
& K_{a b}^{\mu v} V_{v, b}(k)=0 ; \quad k^{v} V_{v, b}(k)=0, \\
& K_{a b}^{\mu v} \equiv g^{\mu v}\left(k^{2} \delta_{a b}-m_{a b}^{2}\right)-k^{\mu} k^{v} \delta_{a b}-i \varepsilon^{\mu v \rho \sigma} \zeta_{\rho} k_{\sigma} N_{a b},
\end{aligned}
$$

selecting out three physical polarizations for massive vector fields. In fact, these three polarizations contribute into the vector field propagators as they couple to conserved fermion currents. The longitudinal polarization $\varepsilon_{L}^{\mu}$ is orthogonal to $k_{\mu}$ and to the CS vector $\zeta_{\mu}$

$$
\varepsilon_{L}^{\mu}=\frac{\zeta^{\mu} k^{2}-k^{\mu}(\zeta \cdot k)}{\sqrt{k^{2}\left((\zeta \cdot k)^{2}-\zeta^{2} k^{2}\right)}}, \quad \varepsilon_{L} \cdot \varepsilon_{L}=-1,
$$

for $k^{2}>0$. The mass of this state remains undistorted. The transversal (circular) polarizations $\varepsilon_{ \pm}^{\mu}$ on the other hand satisfy

$$
K_{v, a b}^{\mu} \varepsilon_{ \pm}^{v} V_{b}(k)=\left(k^{2} \delta_{a b}-m_{a b}^{2} \pm \sqrt{(\zeta \cdot k)^{2}-\zeta^{2} k^{2}} N_{a b}\right) \varepsilon_{ \pm}^{\mu} V_{b}(k)
$$

where $V_{b, \pm}^{\mu}(k)=\varepsilon_{ \pm}^{\mu} V_{b}(k)$. As previously mentioned we restrict ourselves to an isosinglet pseudoscalar background $a(t)$ with nearly constant derivative. Correspondingly the spectrum can be found after the simultaneous diagonalization of matrices $m^{2}, N$ and particularizing to the case $\zeta_{\mu} \simeq(\zeta, 0,0,0)$

$$
\begin{aligned}
& N_{a b} \simeq\left(\begin{array}{ccc}
0 & 0 & 0 \\
0 & \frac{9 g^{2}}{10 e^{2}} & 0 \\
0 & 0 & \frac{9 g^{2}}{10 e^{2}}+1
\end{array}\right) \sim\left(\begin{array}{ccc}
0 & 0 & 0 \\
0 & 1 & 0 \\
0 & 0 & 1
\end{array}\right) \\
& m_{a b}^{2}=m_{V}^{2}\left(\begin{array}{ccc}
0 & 0 & 0 \\
0 & 1 & 0 \\
0 & 0 & 1+\frac{10 e^{2}}{9 g^{2}}
\end{array}\right) \sim\left(\begin{array}{ccc}
0 & 0 & 0 \\
0 & 1 & 0 \\
0 & 0 & 1
\end{array}\right)
\end{aligned}
$$

Finally the dispersion relations for different polarizations are given by,

$$
k_{0}^{2}-\vec{k}^{2}=m_{V}^{2}+\varepsilon \frac{9 g^{2}}{10 e^{2}} \zeta|\vec{k}| \simeq m_{V}^{2}+\varepsilon 360 \zeta|\vec{k}| \equiv m_{V, \varepsilon}^{2},
$$

where the $L, \pm$ polarizations for distorted vector states are accumulated in the discrete variable $\varepsilon=0, \pm 1$. 
Thus in the case of isosinglet pseudoscalar background the massless photons are not distorted when mixed with massive vector mesons. In turn, massive vector mesons split into three polarizations with masses $m_{V,-}^{2}<m_{V, L}^{2}<m_{V,+}^{2}$. This splitting unambiguously signifies local parity breaking as well as breaking of Lorentz invariance due to the time-dependent background. For large enough $|\vec{k}| \geq 10 e^{2} m_{V}^{2} / 9 g^{2} \zeta \simeq m_{V}^{2} / 360 \zeta$ vector meson states with negative polarization become tachyons. However their group velocity remains less than the light velocity [27, [28] provided that $\zeta<20 e^{2} m_{V} / 9 g^{2} \simeq m_{V} / 180 \approx 4.3 \mathrm{MeV}$. For higher values of $\zeta$ the vacuum state becomes unstable , namely, polarization effects give an imaginary part for the vacuum energy. Note that the position of resonance poles for \pm polarized mesons is moving with wave vector $|\vec{k}|$ and therefore they reveal themselves as broadened resonances. The enlargement of the resonant region potentially leads to a substantial enhancement of their contribution to dilepton production away from their nominal vacuum resonance position.

Let's elucidate the ansatz of constant CS background in description of the LPB phenomenon. One can expect the following time dependence of pseudoscalar condensate in heavy ion collisions. At first stage of collision nuclear matter is being compressed and heated (during the time $\tau_{h}$ ), the condensate appears and is supposedly growing. One can extrapolate its field by linear function with a slope $\sim \zeta_{h}$. At the next stage, in the peak of energy density, the pseudoscalar background is nearly constant, i.e. doesn't contribute to LPB. Finally nuclear matter (fireball) is being cooled (during the time $\tau_{c}$ ), the condensate is decreasing and vanish at the freeze-out stage. We again extrapolate it by a linear function with seemingly a different slope $\sim \zeta_{c}$ (of an opposite sign). Thus the parameter $\zeta$ changes its sign, but for the very LPB effect only its modulus is important because changing its sign causes an interchange in polarizations but doesn't affect the splitting of masses for different polarizations. Consequently we can use an average $|\zeta| \simeq\left(\left|\zeta_{h}\right| \tau_{h}+\left|\zeta_{c}\right| \tau_{c}\right) /\left(\tau_{h}+\tau_{c}\right)$ for compression/cooling phases that we actually do. Certainly a more precise treatment must be based on fireball formation/evolution computations which we defer to a future work.

\section{Enhancement of dilepton production for transversal polarizations of vector mesons due to $P$ breaking in medium}

The production rate of dileptons pairs mediated by $\rho$ mesons takes a form similar to the one given in [[13] but with modified propagators due to LPB, according to our previous discussion

$$
\begin{aligned}
& \frac{d N}{d^{4} x d M} \simeq c_{\rho} \Phi\left(M^{2}, m_{l}^{2}\right) \frac{\alpha^{2} \Gamma_{\rho} m_{\rho}^{2}}{3 \pi^{2} g^{2} M^{2}}\left(\frac{M^{2}-4 m_{\pi}^{2}}{m_{\rho}^{2}-4 m_{\pi}^{2}}\right)^{3 / 2} \Theta\left(M^{2}-4 m_{\pi}^{2}\right) \\
& \times \sum_{\varepsilon} \int_{M}^{\infty} d k_{0} \frac{\sqrt{k_{0}^{2}-M^{2}}}{e^{k_{0} / T}-1} \frac{m_{\rho, \varepsilon}^{4}\left(1+\frac{\Gamma_{\rho}^{2}}{m_{\rho}^{2}}\right)}{\left(M^{2}-m_{\rho, \varepsilon}^{2}\right)^{2}+m_{\rho, \varepsilon}^{4} \frac{\Gamma_{\rho}^{2}}{m_{\rho}^{2}}}
\end{aligned}
$$

Finite lepton mass corrections are not shown manifestly in (B.] but accumulated in $\Phi\left(M^{2}, m_{l}^{2}\right)$. A simple thermal average has been included [RQ] but we remark that the temperature $T$ is an effective one that may in fact depend on the range of $M, p_{T}$ and centrality. 
For $\omega$ meson channel of dilepton production a similar expression will be used but with the three pion threshold, its own overall, $c_{\omega}$, constant and kinematic coefficients, characteristic of the dominant $\omega$ decay.

The coefficients $c_{\rho}, c_{\omega}$ parameterize in a phenomenological way the total cross-sections for vector meson creation. Because they are not known with precision in the present setting, particularly their off-shell values, the relative weights are exploited as free parameters in the hadronic 'cocktail' [1], [3], [1]]. This is the procedure used both by NA60 and PHENIX and we shall follow it here too. The usual 'cocktail' contains weights normalized to the peripheral collisions result (roughly agreeing with existing $p p$ and $p$-nucleus data). For semi-central and central collisions, particularly at low $p_{T}$ the $\rho / \omega$ ratio needs to be enhanced (at least) by a factor 1.6 in the case of NA60 [5] or approximately 1.8 in PHENIX [Q, [2]].

A simple thermal average is appropriate only for central collisions and moderate values of $p_{T}$. In order to include a proper description of the $p_{T}$ spectrum a more realistic statistical description would be needed. Nevertheless the general features of the LPB-induced modifications should be already visible in a simplified description to be compared to the experimental data averaged over space-time and momenta for NA60 [B] or acceptance-corrected for PHENIX [ $[\mathbf{G}$ ]. This simple model is appropriate only for central collisions and moderate values of $p_{T}$.

The enhancement due to LPB with respect to the usual 'cocktail' estimates is shown on Fig.t. for $T=150 \mathrm{MeV}$. In the region $300 \mathrm{MeV}<M_{l l}<900 \mathrm{MeV}$ virtual $\rho$ mesons contribute substantially to the production of $l^{+} l^{-}$from $\pi \pi$ fusion. $\omega$ meson decays into three pions are also important but give a subdominant contribution as compared to $\rho$ meson ones, except for the very vicinity of the resonance peak of the $\omega$. In addition, aside of the resonance there is a contribution from the Dalitz decay of the $\eta$ meson and $\omega$ Dalitz decay $\omega \rightarrow \pi e e$ should be taken into account as it is the most important source of enhancement for PHENIX. We will not focus on them here.

The $\rho$ meson has a strong coupling to the $\pi \pi$ channel, and its mean free path $(1.3 \mathrm{fm})$ is essentially shorter than the expected size of the hadronic gas fireball (5-10 fm). Thus in-medium effects for $\rho$ meson must be the most important ones for managing the abnormal dilepton yield.

Note that the $\rho$ mean free path $(1.3 \mathrm{fm})$ is much shorter than the expected size of the hadronic gas fireball $\left(L=5 \div 10 \mathrm{fm}\right.$ ). The life-time of the fireball is comparable $\tau_{F B} \simeq L$. In turn, the mean free path for $\omega$ is 17 times longer, therefore the probability of its decay within the fireball is not as high and the in-medium enhancement due to LPB of the dilepton production is relatively reduced.

Indeed the time spent by a ultra-relativistic resonance of width $\Gamma$ inside the fireball is related to its mean free path $\sim 1 / \Gamma$ in such a medium. Therefore when $\Gamma \tau_{F B} \ll 1$ a tangible suppression of the resonant enhancement $1 / \Gamma^{2}$ arises. Around the distorted resonance peak a crude estimation of this suppression gives $\sim \tau_{F B}^{2} \Gamma^{2}$ in relative units. This suppression affects all long lived particles, such as the ones entering the Dalitz processes, and it is also relevant to $\omega$ meson decays away from the vacuum peak. It represents the ultimate reason why it is unnecessary to include the $\phi$ vector resonance in the discussion. Neglecting this reduction the amplification of dilepton yield is presented on Fig. [.

Nevertheless the resonance peak is mostly saturated by $\omega$ decays and the net effect is depicted on Fig.6. Comparison with the results from PHENIX indicate that a value for $\zeta$ between 1 and 2 $\mathrm{MeV}$ provides enough enhancement in this region to explain the dilepton excess. 


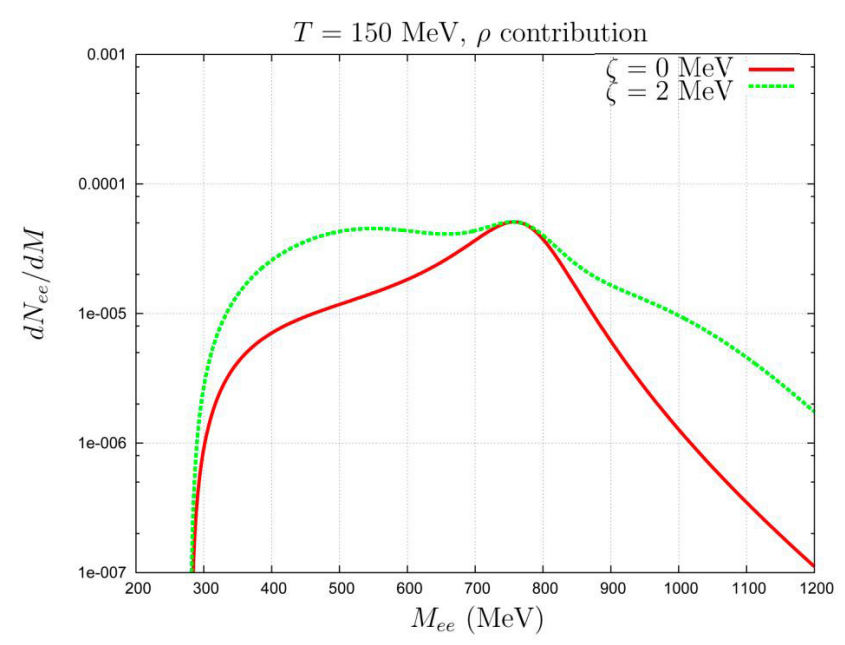

Figure 4: The $\rho$ meson contribution into the dilepton production is shown for parity symmetric nuclear matter $\zeta=0$ and for local parity breaking with $\zeta=2 \mathrm{MeV}$. The units of vertical axis are normalized on those for the PHENIX experimental data [ $[\mathbf{D}]$.

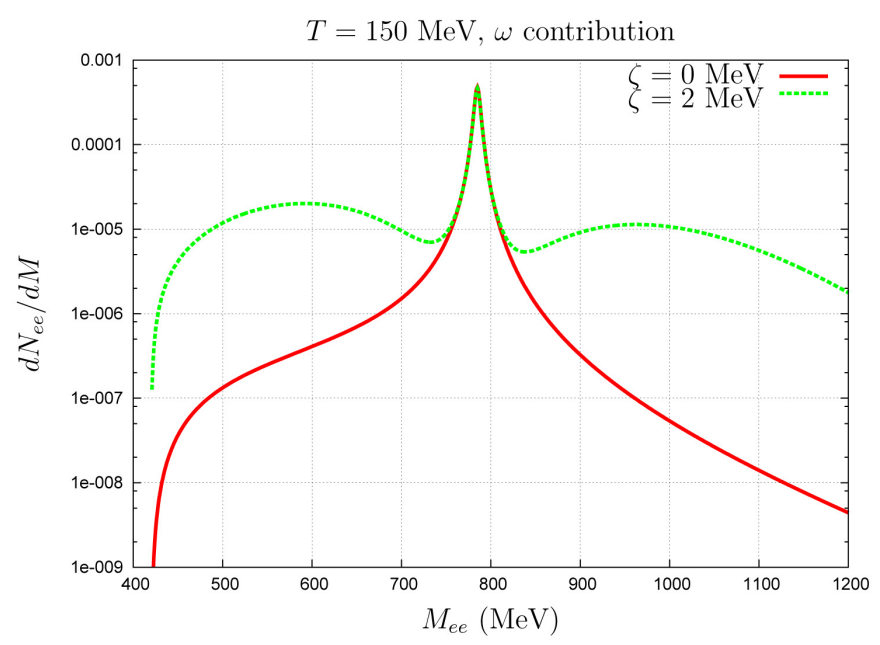

Figure 5: The $\omega$ meson contribution into the dilepton production is shown for parity symmetric nuclear matter $\zeta=0$ and for local parity breaking with $\zeta=2 \mathrm{MeV}$. The limit of very large fireball is taken.

At lower invariant masses $<300 \mathrm{MeV}$ the Dalitz processes $\pi_{0} \rightarrow \gamma e^{+} e^{-}, \eta, \eta^{\prime} \rightarrow \gamma e^{+} e^{-}$or $\eta, \eta^{\prime} \rightarrow \gamma \mu^{+} \mu^{-}$nearly saturate the $l^{+} l^{-}$production. The Dalitz process $\omega \rightarrow \pi l^{+} l^{-}$is also of importance. As before, for an isosinglet background only the spectral densities of virtual $\rho$ and $\omega$ states are modified by the pseudoscalar background resulting from LPB. In this case the analog of the Kroll-Wada formula [B3] describes the partial decay widths from the $L, \pm \rightarrow \varepsilon=0, \pm 1$ 


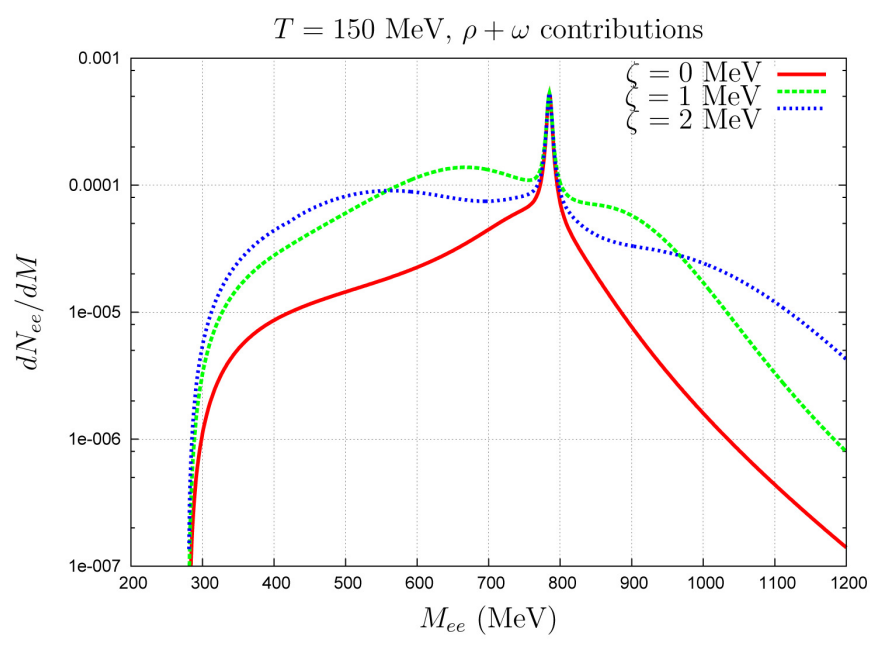

Figure 6: The net meson contribution into the dilepton production is shown for parity symmetric nuclear matter $\zeta=0$ and for local parity breaking with $\zeta=1,2 \mathrm{MeV}$. The normalization of the $\rho+\omega$ peak is chosen on the PHENIX data.

polarizations for distorted vector states

$$
\begin{aligned}
\frac{d N_{e e}}{d M d^{4} x d^{3} q} \simeq \sum_{V, P} c_{P} \frac{\alpha^{3} m_{P}^{3}}{144 \pi^{4} f_{P}^{2} M}\left(1-\frac{M^{2}}{m_{P}^{2}}\right)^{3} \sum_{\varepsilon}\left|F\left(M, m_{V, \varepsilon}\right)\right|^{2} \equiv \frac{d \Gamma_{\gamma e e}}{d M} \\
F\left(M, m_{V, \varepsilon}\right) \equiv\left[\left(1-\frac{M^{2}}{m_{V, \varepsilon}^{2}}\right)^{2}-i \frac{\Gamma_{V e e}}{m_{V}}\right]^{-1},
\end{aligned}
$$

where for massive vector mesons (2.10)

$$
m_{V, \varepsilon}^{2} \simeq M_{\rho}^{2}+\varepsilon 360 \zeta|\vec{k}|, m_{V}^{2} \equiv m_{V, 0}^{2} \simeq M_{\rho}^{2}
$$

and

$$
\Gamma_{\rho e e} / m_{\rho} \simeq 10^{-5} ; \Gamma_{\omega e e} / m_{\omega} \simeq 0.8 \cdot 10^{-6} .
$$

The coefficients $c_{P}$ correct the ratio of $\pi, \eta$ two-photon decays, $c_{\eta}: c_{\pi} \simeq 4.3$ [B3] and also include the production rate per unit volume of pseudoscalar mesons $[\square]$. This production rate must be also averaged over the thermal distribution of pseudoscalar mesons. The positions of resonances $m_{V, \pm}^{2}$ move with the wave vector $\vec{k}$ and therefore convolution with thermal distribution makes them broader. In Eq.(13.3) the enhancement is calculated for very large nuclear matter fireballs and it is definitely overestimated. As the meson resonance life time is essentially larger than the collision time $\tau \sim 5 \div 10 \mathrm{fm}$ one should expect a strong finite volume suppression. Still one could expect a visible enhancement $\sim \tau^{2} m_{V, \varepsilon}^{2}>1$ for $m_{V, \varepsilon}>100 \mathrm{MeV}$. This estimate is valid for $\Gamma_{V e e} \tau \ll 1$. An additional reduction is caused by convolution with the thermal distribution.

When $\hat{\zeta} \neq 0$ and its isospin content is arbitrary $\hat{\zeta}=A \mathbf{I}+B \tau_{3}$ the photons of "+" polarization behave as narrow resonances [[D] with the width $\Gamma_{\gamma} \simeq \alpha \zeta / 3$ and decay into $l^{+} l^{-}$above the threshold at $|\vec{k}|>4 m_{l}^{2} / \zeta$. This mechanism of enhancement could be dominant for production of $e^{+} e^{-}$ at relatively low invariant masses $<300 \mathrm{MeV}$ when the Dalitz processes $\pi_{0} \rightarrow \gamma e^{+} e^{-}, \eta \rightarrow \gamma e^{+} e^{-}$ 


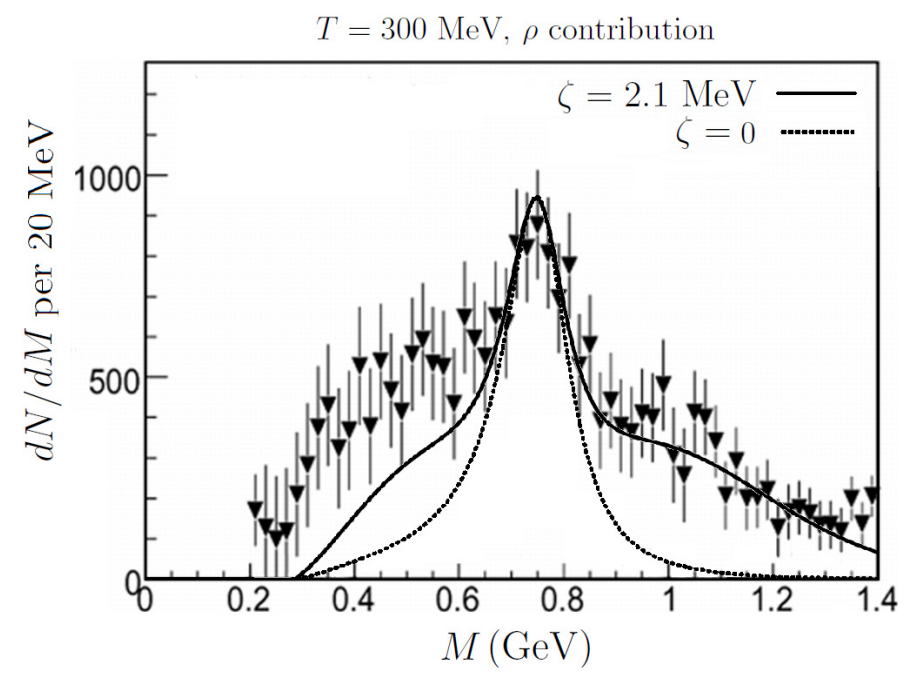

Figure 7: The $\rho$ meson contribution into the dilepton production is shown for parity symmetric nuclear matter $\zeta=0$ and for local parity breaking with $\zeta=2 \mathrm{MeV}$ compared to the NA60 measurement for central collisions and all $p_{T}$. Both contributions are normalized to produce the same on-shell cross-section for $\rho$ meson production, which is fitted to the experiment. The effective temperature is $300 \mathrm{MeV}$.

saturate the $e^{+} e^{-}$production. However for dimuons the threshold is too high to make photon decays of any importance for dimuon production.

\section{Fitting the PHENIX and NA60 data on dilepton excess}

NA60 has obtained accurate results for the $\rho$ spectral function[可] by measuring the $\mu^{+} \mu^{-}$ spectrum with unprecedent precision and by carefully subtracting the contributions from the 'cocktail' except the $\rho$ itself. The corresponding data for central collisions is shown in Fig.ర along with the contribution from the 'cocktail' scaled by the 2.3 factor and using $m_{\rho}=750 \mathrm{MeV}$. The 'cocktail' $\rho$ can be obtained from (B. $($ d) by setting $\zeta=0$ and adjusting the effective temperature to $T \simeq 300 \mathrm{MeV}$ [B] $]$. However, even after optimization of the constant to reproduce central $\rho$ production, the agreement of the data with the 'cocktail' $\rho$ is poor reflecting the long standing problem of the insufficient dilepton yield. In the same figure we show, after adjusting the scale to reproduce the $\rho$ peak, the result for $\zeta \simeq 2 \mathrm{MeV}$. Clearly the agreement is much better, particularly on the right of the $\rho$ peak. On the left of the resonance there is a noticeable discrepancy for $M<650 \mathrm{MeV}$ approximately; experimental points seem to be shifted upwards by a small constant amount with respect to the prediction from LPB. It is revealing that the upper kinematical limit for the Dalitz process $\omega \rightarrow \mu^{+} \mu^{-} \pi^{0}$ is $643 \mathrm{MeV}$.

Important contributions to dilepton production at lower invariant masses are the Dalitz processes $\pi_{0} \rightarrow \gamma e^{+} e^{-}, \eta \rightarrow \gamma l^{+} l^{-}$, almost saturating the $l^{+} l^{-}$production in the $M<300 \mathrm{MeV}$ region, and $\omega \rightarrow l^{+} l^{-} \pi^{0}$. The latter has a partial lepton width nearly identical to the one of $\rho \rightarrow l^{+} l^{-}$and thus expected to show a similar behavior. The Kroll-Wada formula[[3]] of the type (B.3) includes the contribution of vector mesons to the previous Dalitz processes and it remains valid in the case of LPB provided that we replace the vector meson masses by the values in (R.TO) according to the intermediate meson polarization $(L, \pm)$. We have checked that this contribution shows an en- 


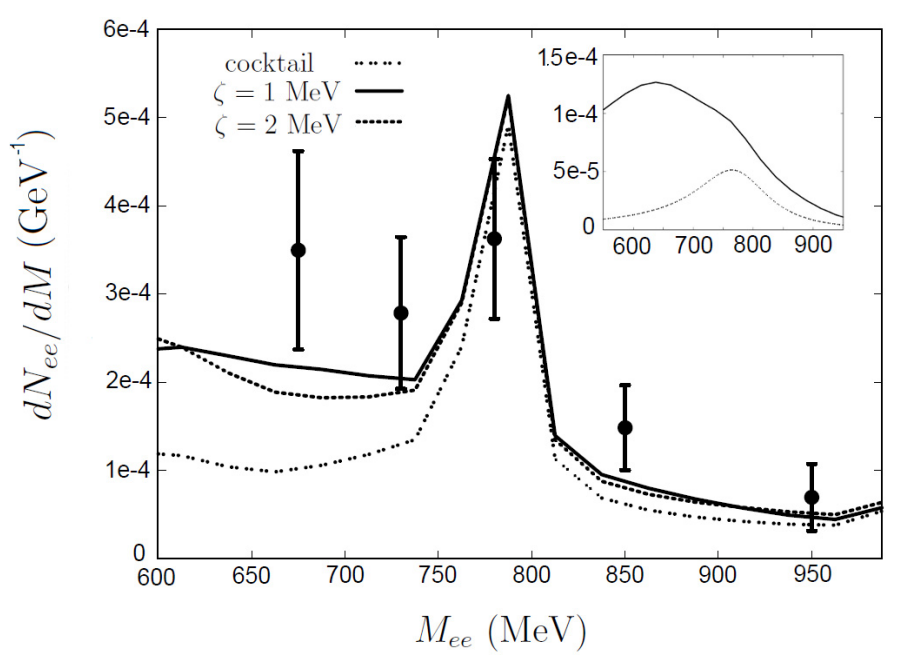

Figure 8: The meson contribution to dilepton production is shown for parity symmetric nuclear matter $\zeta=0$ (discontinuous line) and for local parity breaking with $\zeta=1,2 \mathrm{MeV}$ (solid line and dotted line, respectively) compared to the PHENIX measurements for minimum bias, all $p_{T}$ events. The $\zeta=0$ line is just the 'cocktail' contribution. The $\zeta \neq 0$ results from enhancing the $\rho / \omega$ ratio by a factor 1.8 without changing the $\omega$ normalization w.r.t. the hadronic 'cocktail'. The $\omega$ contribution itself includes a $20 \%$ of LPB contribution $(\zeta=1)$ and $80 \%$ pure 'cocktail' $(\zeta=0)$ to partially account for medium effects. A reasonable fit is obtained for $T=110 \mathrm{MeV}$ (compatible with PHENIX estimates for low $p_{T}$ ) and $\zeta=1 \mathrm{MeV}$. Data for $M<650 \mathrm{MeV}$ is not shown as the contribution from the Dalitz processes has not been included. Inset: the rho spectral function for $\zeta=1 \mathrm{MeV}$ is compared to the cocktail one with the same assumptions.

hancement but this and other hadronic processes relevant for dilepton production will be discussed elsewhere. Indeed NA60 has been able to identify the individual contributions from $\eta$ and $\omega$ Dalitz decays and found an enhancement, particularly for the latter[B]].

One should stress that the fit presented saturated almost all the effect of enhancement by normalizing properly the position of $\rho$ resonance. However a more conventional thermal broadening must also contribute and may improve the quality of prediction in lower wing. This program elaborated in [[3] is of importance to be taken into account in order to establish the interplay between purely thermal and LPB effects and it is in progress.

Moving now to the PHENIX $e^{+} e^{-}$data[ [प, [34] we observe that it has a much poorer precision and a numerical subtraction of the 'cocktail' to determine different individual contributions is meaningless. For low values of $p_{T}$ and for central collisions the effective temperature quoted by PHENIX lies in the region $100 \div 150 \mathrm{MeV}$ for the range of invariant masses under consideration. Based on the standard 'cocktail' we apply the normalization factor 1.8 already mentioned in order to approximately account for the correct $\rho / \omega$ ratio and use Eq. (B.]) with $\zeta \neq 0$ to include the effects of LPB. Fig. 8 shows the predicted $e^{+} e^{-}$yield for $\zeta=1 \mathrm{MeV}$ which is close to the optimal fit. The precision of the data does not allow for such clear cut conclusions as in the case of NA60 but it is evident that LPB noticeably improves the agreement to the data as compared to the 'cocktail'. We would like to emphasize the extraordinary simplicity of the approach presented here. The fits presented use the values (effective temperatures, normalizations, etc. ) quoted by the experiment themselves. The only free parameter is $\zeta$, which is expected to depend on the characteristics of 
the collision. It should also be said clearly that the presence of LPB does not preclude other many body or in-medium corrections[[13], as long as they do not represent double counting.

\section{Conclusions and perspectives in discovery of local parity breaking}

We now summarize the signatures and outline possible searches of local parity breaking. Polarization: Dileptons produced for values of the invariant mass above and below the $\rho+\omega$ pole are predominantly of opposite circular polarizations. Thus one could search for a asymmetries among longitudinal and transverse polarization for different $M$ in event-by-event measurements. These measurements may reveal in an unambiguous way the existence of parity breaking.

Distorted photons: At low energies massive vectors dominate the amplitudes but photons should also reveal a distortion induced by LPB. Those with ' + ' polarization exhibit different momentum thresholds $\sim 4 m_{l}^{2} / \zeta$ to show a resonant behavior for different dilepton species[ㅁ]]. Note that finite size suppression is relevant for photons.

The nature of the condensate: Mixing of photons with vector mesons is sensitive to isospin of pseudoscalar condensate and therefore the fraction of distorted photon decays helps to disentangle its isospin content. To summarize, in a time-dependent pseudoscalar background massless photons of ' + ' polarization and massive vector mesons behave as broadened resonances after averaging over thermal distribution.

In conclusion, for an isosinglet time-dependent pseudoscalar background the vector mesons $\rho$ and $\omega$ propagators are distorted. We have computed this effect and found that it naturally tends to produce an overabundance of dilepton pairs in the $\rho+\omega$ resonance region. The modified $\rho$ spectral function shows features very similar to the ones measured by NA60 in dimuon events. The PHENIX data are much better described by this mechanism than by adjusting the standard hadronic 'cocktail' in the $\rho+\omega$ resonance region. At lower invariant masses the Dalitz processes $\pi^{0} \rightarrow \gamma e^{+} e^{-}, \eta \rightarrow \gamma l^{+} l^{-}$and $\omega \rightarrow l^{+} l^{-} \pi^{0}$ saturate the hadronic contribution to $l^{+} l^{-}$production and are enhanced by the LPB-induced modifications on the vector meson propagators; detailed results are deferred to a future publication. The only free parameter is $\zeta$, characterizing the time variation of the pseudoscalar condensate. A good fit to the data is obtained for natural values of $\zeta$. The possibility that the LPB condensate is an isotriplet or an admixture of isotriplet and isosinglet has been discussed. Experimental signals of the manifestation of LPB in heavy ion collisions have been suggested. Thus local parity breaking seems capable of explaining in a natural way the PHENIX/CERES/NA60 'anomaly' and searching for its manifestation in dilepton production represents an interesting experimental challenge.

We acknowledge the financial support from projects FPA2007-66665, 2009SGR502, CPAN (Consolider CSD2007-00042) and FLAVIANET. A.\& V. Andrianov are supported also by Grants RFBR 09-02-00073-a and 10-02-00881-a and by SPbSU grant 11.0.64.2010. We thank D. d'Enterria and J. Casalderrey for discussions and useful remarks.

\section{References}

[1] A.B. Migdal, Zh. Eksp. Teor. Fiz. 61 (1971) 2210 [Sov. Phys. JETP 36 (1973) 1052]; R.F. Sawyer, Phys. Rev. Lett. 29 (1972) 382; 
D.J. Scalapino, Phys. Rev. Lett. 29 (1972) 386;

G. Baym, Phys. Rev. Lett. 30 (1973) 1340;

A.B. Migdal, O.A. Markin and I.N. Mishustin, Sov. Phys. JETP,39 (1974) 212.

[2] T. Shamsunnahar, S. Saha, K. Kabir and L.M. Nath, J. Phys. G: Nucl. Pan. Phys. 17 (1991) 887;

A. Akmal, V.R. Pandharipande, Phys. Rev. C56(1997) 2261.

[3] A. Andrianov and D. Espriu, Phys. Lett. B 663 (2008) 450;

A. Andrianov, V. Andrianov and D. Espriu, Phys. Lett. B 678 (2009) 416.

[4] D. Kharzeev, R.D. Pisarski and M.H.G. Tytgat, Phys. Rev. Lett. 81 (1998) 512;

K. Buckley, T. Fugleberg, and A. Zhitnitsky, Phys. Rev. Lett. 84 (2000) 4814;

D. Kharzeev, Phys. Lett. B 633 (2006) 260;

D. Kharzeev and A. Zhitnitsky, Nucl. Phys. A 797 (2007) 67;

D.E. Kharzeev, L.D. McLerran and H.J. Warringa, Nucl. Phys. A 803 (2008) 227.

[5] M. Masera, (HELIOS/3 Collaboration), Nucl. Phys. A 590 (1995) 103c;

G. Agakichiev et al.(CERES Collaboration), Eur. Phys. J. C 4 (1998) 231.

[6] R. Arnaldi et al.(NA60 Collaboration), Phys. Rev. Lett. 96, 162302 (2006).

[7] PHENIX Collaboration (A. Adare et al.), Phys. Rev. C 81, 034911 (2010).

[8] G. Agakichiev et al. (HADES Collab.), Phys. Rev. Lett. 98, 052302 (2007) ; Phys.Lett. B 663 (2008) 43.

[9] K.O. Lapidus and V.M. Emel'yanov, Phys. Part. Nucl. 40 (2009) 29.

[10] I. Tserruya, 0903.0415 [nucl-ex].

[11] G. Agakichiev et al. (HADES Collab.), Phys.Lett. B 690 (2010) 118.

[12] G.E. Brown and M. Rho, Phys. Rev. Lett. 66 (1991) 2720.

[13] R. Rapp and J. Wambach, Adv. Nucl. Phys. 25 (2000) 1;

W. Liu, R. Rapp, Nucl. Phys. A 796 (2007) 101;

H. van Hees and R. Rapp, Nucl. Phys. A 806 (2008) 339.

[14] W. Cassing and E. Bratkovskaya, Nucl. Phys. A 807 (2008) 214;

E.L. Bratkovskaya, W. Cassing and O. Linnyk, Phys. Lett. B 670 (2009) 428.

[15] K. Dusling and I. Zahed, Nucl. Phys. A 825 (2009) 212.

[16] A. Andrianov, V. Andrianov, D. Espriu and X. Plannels, arXiv:1010.4688 [hep-ph].

[17] P. Buividovich, M. Chernodub, E. Luschevskaya and M. Polikarpov, Phys. Rev. D 80, 054503 (2009) .

[18] B. I. Abelev et al. [STAR Collaboration], Phys. Rev. Lett. 103, 251601 (2009) ;

S.A. Voloshin, J. Phys. Conf. Ser. 230, 012021 (2010) .

[19] A. Gorsky and M.B. Voloshin, 1006.5423 [hep-th] .

[20] A.A. Andrianov, D. Espriu, P. Giacconi and R. Soldati, JHEP 0909, 057 (2009) ; A.A. Andrianov, D. Espriu, F. Mescia and A. Renau, Phys. Lett. B 684 (2010) 101.

[21] J.J. Sakurai, Ann. of Physics 11 (1960) 1; Currents and Mesons, The University of Chicago Press, Chicago 1969.

[22] N. Kaiser and U.-G. Meissner, Nucl. Phys. A 519 (1990) 671;

E. Truhlik, J. Smejkal and F.C. Khanna, Nuclear Physics A 689(2001) 741. 
[23] O. Dumbrais et al., Nucl. Phys. B 216 (1983) 277.

[24] F. Klingl, N. Kaiser and W. Weise, Z. Phys. A 356 (1996) 193.

[25] K. Nakamura et al. (Particle Data Group), J. Phys. G 37 , 075021 (2010).

[26] T. Feldmann, P. Kroll and B. Stech, Phys. Rev. D 58, 114006 (1998); Y.N. Klopot, A.G. Oganesian and O.V. Teryaev, arXiv:0911.0180 [hep-ph] .

[27] J. Alfaro, A.A. Andrianov, M. Cambiaso, P. Giacconi and R. Soldati, Int. J. Mod. Phys. A 25 (2010) 3271.

[28] A.A. Andrianov and R. Soldati, Phys. Lett. B 435 (1998) 449.

[29] E.L. Feinberg, Nuovo Cim. A 34 (1976) 391;

L. McLerran and T. Toimela, Phys. Rev. D 31 (1985) 545.

[30] R. Arnaldi et al [NA60 Collaboration], Phys. Lett. B 677 (2009) 260.

[31] R. Arnaldi et al. [NA60 Collaboration], Eur. Phys. J. C 61 (2009) 711.

[32] V.L. Rykov, private communication.

[33] L.G. Landsberg, Phys. Rep. 128 (1985) 301.

[34] Numerical data extracted from http://www.phenix.bnl.gov/phenix/WWW/info/data/ppg088_data.html/fig26.txt 